\title{
A shape optimization algorithm for the minimum drag problem in Stokes flow*
}

\author{
A. P. Suetov \\ Institute of Mathematics and Mechanics, Russian Academy of Science \\ 620219 Ekaterinburg, S.Kovalevskoi Str. 16, Russia. \\ Phone: (3432)493143. Fax: (3432)442581. \\ $E$-mail: suetov@odu.imm.intec.ru
}

\begin{abstract}
This paper deals with the theory of shape (or domain) optimization. A model minimum drag problem in Stokes flow is considered. An algorithm for computation of a solution of the extended problem is given. The algorithm is based on reduction of the shape optimization problem to a family of coefficient optimization problems.
\end{abstract}

\section{Keywords}

Shape optimization, generalized solutions, computation algorithm

\section{INTRODUCTION AND NOTATIONS}

In the present paper a problem related to the theory of optimal shape design (Banichuk, 1983; Pironneau, 1984) is considered. From the mathematical point of view the shape is a subset in $\mathbf{R}^{\mathbf{n}}$. The main feature of the problem is that the cost functional depends on the set by via the solution of the boundary value problem. We apply the approach used in (Suetov, 1994). According to this approach in Section 2 the known shape optimization problem is formulated and a definition of its generalized solutions is given. In Section 3 an extended shape optimization problem is formulated. In Section 4 a coefficient optimization problem is considered and some connections with the problems of Sections 2, 3 are established. In Section 5 an algorithm for approximate shape optimization is proposed. In this algorithm the problem of determination of an optimal shape is replaced by a family of coefficient optimization problems.

We will use the following notation:

- $\partial M$ is the boundary of the set $M \subset \mathbf{R}^{3}$;

- meas $(M)$ is the Lebesgue measure of a set $M \subset \mathbf{R}^{3}$;

\footnotetext{
*The research described in this publication was made possible in part by grant NMD300 from Interna-
} tional Science Foundation and Russian Government 
- $\mathrm{W}_{2}^{1}(\Omega)$ is the Sobolev space $\left(\Omega \subset \mathbf{R}^{3}\right.$ is open), $\mathrm{W}_{2}^{1}(\Omega)=\left(\mathrm{W}_{2}^{1}(\Omega)\right)^{3}$ (Adams, 1975);

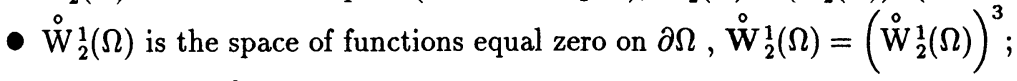

- $V(\Omega)=\left\{\mathbf{u} \in \stackrel{\circ}{\mathbf{W}}_{2}^{\mathbf{1}}(\Omega): \operatorname{div} \mathbf{u}=0\right\}$ (Temam, 1979) ;

- for $\mathbf{u}=\left(u_{1}, u_{2}, u_{3}\right) \in \mathbf{W}_{2}^{1}(\Omega)$ let $\nabla \mathbf{u}=\left(\frac{\partial u_{i}}{\partial x_{j}}\right), \nabla \mathbf{u} \nabla \mathbf{v}=\sum_{i, j=1}^{3} \frac{\partial u_{i}}{\partial x_{j}} \frac{\partial v_{i}}{\partial x_{j}}$;

- $\Delta$ is the Laplace operator, $\Delta \mathbf{u}=\left(\Delta u_{1}, \Delta u_{2}, \Delta u_{3}\right)$;

- $\|\mathrm{f} \mid \mathrm{Y}\|$ is the norm of a function $\mathrm{f}$ in a space $\mathrm{Y}$.

\section{A SHAPE OPTIMIZATION PROBLEM AND ITS GENERALIZED SOLUTIONS}

We consider a problem of finding the shape of the body of unit volume which produces minimum drag when moving slowly through a viscous fluid at constant speed (Watson, 1971; Pironneau, 1973).

Let $\mathbf{T}$ be a bounded open set in $\mathbf{R}^{3}, \operatorname{meas}(\mathbf{T})>1, \partial \mathbf{T}=\Gamma=\Gamma_{1} \cup \Gamma_{2}, \Gamma_{1}$ is parallel to the plan $O x_{2} x_{3}$ and $\Gamma_{2}$ is parallel to the axis $O x_{1}$. Let $\mathbf{B}$ be a closed subset of $\mathbf{T}, \partial \mathbf{B}=S$ (see Figure 1).
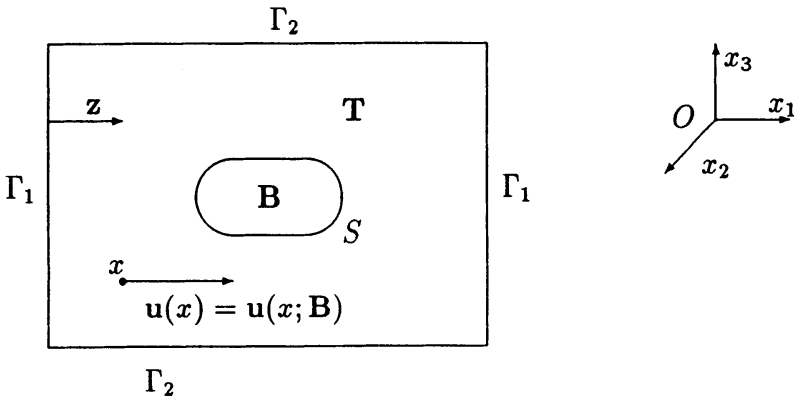

Figure 1 A body in the flow.

Let us consider the following boundary value problem: $\mathbf{u}=\left(u_{1}, u_{2}, u_{3}\right)$,

$\triangle \mathbf{u}=\nabla p, \quad \operatorname{div} \mathbf{u}=\mathbf{0} \quad$ in $\quad \mathbf{T} \backslash \mathbf{B} ;\left.\quad \mathbf{u}\right|_{\Gamma=\mathbf{z}}=(1,0,0),\left.\quad \mathbf{u}\right|_{S}=0$

We will denote by $\mathbf{u}(\mathbf{B})$ the weak solution of this problem. As the cost functional we accept the value which is proportional to the rate of energy dissipated by the fluid flow $\mathbf{u}(\mathbf{B})$ :

$J(\mathbf{B})=F(\mathbf{u}(\mathbf{B}))=\int_{\mathrm{T}} \frac{1}{2} \sum_{i, j=1}^{3}\left(\frac{\partial u_{i}}{\partial x_{j}}+\frac{\partial u_{j}}{\partial x_{i}}\right)^{2} \mathrm{dx}$.

We define the class of admissible sets as

$\Phi=\{\mathbf{B}: \mathbf{B} \subset \mathbf{T}, \mathbf{B}$ is closed, meas $(\mathbf{B}) \geq 1\}$. 
Problem 1. It is required to find an admissible set $\mathrm{B}^{*} \in \Phi$, minimizing the functional $J(\mathrm{~B})$ over the class of sets $\Phi$ :

$$
\mathbf{B}^{*}=\inf _{\mathbf{B} \in \Phi} J(\mathbf{B}) \text {. }
$$

The question of existence of a solution of Problem 1 is not trivial (Osipov and Suetov, 1984; Pironneau, 1984), therefore the following definition is reasonable.

Definition. A generalized solution of the shape optimization problem is a weak limit point in $\mathbf{W}_{2}^{1}(\mathbf{T})$ of a sequence $\left\{\mathbf{u}\left(\mathbf{B}_{i}\right)\right\}$, where $\left\{\mathbf{B}_{i}\right\}$ is a minimizing sequence for Problem 1 .

\section{Statement 1 There exists a generalized solution of Problem 1.}

Proof. A sequence $\left\{\mathbf{u}\left(\mathbf{B}_{i}\right)\right\}, \mathbf{B}_{i} \subset \mathbf{T}$, is bounded in space $\mathbf{W}_{2}^{1}(\mathbf{T})$ (Temam, 1979), therefore it has a weak limit point.

Let $X=\left\{\mathbf{u} \in \mathbf{W}_{2}^{1}(\mathbf{T}): \operatorname{div} \mathbf{u}=0,\left.\quad \mathbf{u}\right|_{\Gamma}=\mathbf{z}\right\}$ and $X(\mathbf{B})=\left\{\mathbf{u} \in \mathbf{W}_{\mathbf{2}}^{1}(\mathbf{T}): \operatorname{div} \mathbf{u}=\right.$ $\left.0,\left.\quad \mathbf{u}\right|_{\mathbf{B}}=0,\left.\quad \mathbf{u}\right|_{\Gamma=\mathbf{z}}\right\}$. It is clear that $X=X(\emptyset), X(\mathbf{B}) \subset X \subset \mathbf{W}_{2}^{1}(\mathbf{T}),(X(\mathbf{B})-\mathbf{z}) \subset$ $V(\mathbf{T})$. Let us recall that $\mathbf{u}(\mathbf{B})$ is the solution of the variational identity

$\mathbf{u} \in X(\mathbf{B}) \quad \forall \mathbf{v} \in V(\mathbf{T} \backslash \mathbf{B}) \quad \int_{\mathbf{T}} \nabla \mathbf{u} \cdot \nabla \mathbf{v d x}=0$.

and, equivalently, is the solution of the minimization problem

$E(\mathbf{u}(\mathbf{B}))=\min _{\mathbf{u} \in X(\mathbf{B})} E(\mathbf{u}), \quad E(\mathbf{u})=\int_{\mathbf{T}}(\nabla \mathbf{u})^{2} \mathrm{~d} \mathbf{x}$

(Temam, 1979).

Lemma 1 For every $\mathbf{u} \in X$ we have $F(\mathbf{u})=E(\mathbf{u})$.

Proof. Let us transform the energy dissipation formula

$\frac{1}{2} \sum_{i, j=1}^{3}\left(\frac{\partial u_{i}}{\partial x_{j}}+\frac{\partial u_{j}}{\partial x_{i}}\right)^{2}=\sum_{i, j=1}^{3}\left(\frac{\partial u_{i}}{\partial x_{j}}\right)^{2}+\sum_{i, j=1}^{3} \frac{\partial u_{i}}{\partial x_{j}} \frac{\partial u_{j}}{\partial x_{i}}=$

$\sum_{i, j=1}^{3}\left(\frac{\partial u_{i}}{\partial x_{j}}\right)^{2}+\sum_{j=1}^{3} \frac{\partial}{\partial x_{j}}\left(\sum_{i=1}^{3} u_{i} \frac{\partial u_{j}}{\partial x_{i}}\right)-\sum_{i=1}^{3} u_{i} \frac{\partial}{\partial x_{i}} \sum_{j=1}^{3} \frac{\partial u_{j}}{\partial x_{j}}$

In virtue to div $u=0$ the third term equals zero. Let us apply Green's formula to the integral of the second term

$\int_{\mathbf{T} \backslash \mathbf{B}} \sum_{j=1}^{3} \frac{\partial}{\partial x_{j}}\left(\sum_{i=1}^{3} u_{i} \frac{\partial u_{j}}{\partial x_{i}}\right) \mathrm{dx}=\int_{\partial(\mathbf{T} \backslash \mathbf{B})} \sum_{j=1}^{3}\left(\sum_{i=1}^{3} u_{i} \frac{\partial u_{j}}{\partial x_{i}}\right) n_{j} \mathrm{ds}$

Let us recall $\partial(\mathbf{T} \backslash \mathbf{B})=S \cup \Gamma_{1} \cup \Gamma_{2}$, The condition $\left.\mathbf{u}\right|_{S}=0$ gives $\int_{S} \sum_{i, j=1}^{3} u_{i} \frac{\partial u_{j}}{\partial x_{i}} n_{j} \mathrm{ds}=0$. 
Since $\Gamma_{1}$ is parallel to $O x_{2}, O x_{3}$ and $\left.\mathbf{u}\right|_{\Gamma_{1}}=\mathbf{z}$ we see that $\left.\frac{\partial u_{i}}{\partial x_{2}}\right|_{\Gamma_{1}}=0,\left.\frac{\partial u_{i}}{\partial x_{3}}\right|_{\Gamma_{1}}=0$ for all $i, j$. Equalities $\frac{\partial u_{2}}{\partial x_{2}}=0, \frac{\partial u_{3}}{\partial x_{3}}=0$ and $\operatorname{div} \mathbf{u}=0$ imply $\left.\frac{\partial u_{1}}{\partial x_{1}}\right|_{\Gamma_{1}}=0$. From $\left.\mathbf{u}\right|_{\Gamma_{1}}=(1,0,0)$ and $\left.\mathbf{n}\right|_{\Gamma_{1}}=\left(n_{1}, 0,0\right)$ we have $\sum_{i, j=1}^{3} u_{i} \frac{\partial u_{j}}{\partial x_{i}} n_{j}=u_{1} \frac{\partial u_{1}}{\partial x_{1}} n_{1}=0$ at every point of $\Gamma_{1}$. Thus $\int_{\Gamma_{1}} \sum_{i, j=1}^{3} u_{i} \frac{\partial u_{j}}{\partial x_{i}} n_{j} \mathrm{ds}=0$.

By the definition of $\mathbf{T}$ the surface $\Gamma_{2}$ is parallel to $O x_{1}$ and $\left.\mathbf{u}\right|_{\Gamma_{2}}=\mathbf{z}$, hence $\frac{\partial u_{j}}{\partial x_{1}}=0$ for all $j$. From $\left.\mathbf{u}\right|_{\Gamma_{2}}=(1,0,0)$ and $\left.\mathbf{n}\right|_{\Gamma_{2}}=\left(0, n_{2}, n_{3}\right)$ it follows that $\sum_{i, j=1}^{3} u_{i} \frac{\partial u_{j}}{\partial x_{i}} n_{j}=$ $u_{1}\left(\frac{\partial u_{2}}{\partial x_{1}} n_{2}+\frac{\partial u_{3}}{\partial x_{1}} n_{3}\right)=0$ at every point of $\Gamma_{2}$. Hence

$\int_{\Gamma_{2}} \sum_{i, j=1}^{3} u_{i} \frac{\partial u_{j}}{\partial x_{i}} n_{j} \mathrm{ds}=0, \quad \int_{\mathbf{T} \backslash \mathbf{B}} \sum_{i, j=1}^{3} \frac{\partial u_{i}}{\partial x_{j}} \frac{\partial u_{j}}{\partial x_{i}} \mathrm{dx}=\int_{\partial(\mathbf{T} \backslash \mathbf{B})} \sum_{i, j=1}^{3} u_{i} \frac{\partial u_{j}}{\partial x_{i}} n_{j} \mathrm{ds}=0$

and

$\forall \mathbf{u} \in X \quad F(\mathbf{u})=\int_{\mathbf{T} \backslash \mathbf{B}} \frac{1}{2} \sum_{i, j=1}^{3}\left(\frac{\partial u_{i}}{\partial x_{j}}+\frac{\partial u_{j}}{\partial x_{i}}\right)^{2} \mathrm{~d} \mathbf{x}=\int_{\mathbf{T} \backslash \mathbf{B}} \sum_{i, j=1}^{3}\left(\frac{\partial u_{i}}{\partial x_{j}}\right)^{2} \mathrm{dx}=E(\mathbf{u})$.

Remark 1 It is clear from lemma 1 and (3) that $J(\mathbf{B})=E(\mathrm{u}(\mathbf{B}))$ and

$\inf _{\mathbf{B} \in \Phi} J(\mathbf{B})=\inf _{\mathbf{B} \in \Phi} \inf _{\mathbf{u} \in X(\mathbf{B})} E(\mathbf{u})=\inf _{\mathbf{u} \in U_{1}} E(\mathbf{u}), \quad U_{1}=\bigcup_{\mathbf{B} \in \Phi} X(\mathbf{B})$

This remark is similar to the variational principle from (Watson, 1971).

\section{AN AUXILIARY OPTIMIZATION PROBLEM}

Let us formulate an optimization problem similarly to (Pironneau, 1984, ch.3.2.2).

Problem 2. Let $U=\{\mathbf{u} \in X: \operatorname{meas}(x \in \mathbf{T}:|\mathbf{u}(x)|=0) \geq 1\}$. It is required to find a function $\mathbf{u}^{*} \in U$ minimizing the functional $E(\mathbf{u})$ over the class $U$ :

$$
E\left(\mathbf{u}^{*}\right)=\min _{\mathbf{u} \in U} E(\mathbf{u})
$$

Statement 2 There exists a solution of Problem 2. A weak limit point of a minimizing sequence of Problem 2 is a strong limit point of the sequence.

Proof. The existence follows from the coercivity and weakly lower semicontinuity of $E(\mathbf{u})$ on $\stackrel{\circ}{W}_{2}^{1}(T)$, from compactness of the imbedding $\mathbf{W}_{2}^{1}(\mathbf{T})$ into $\mathbf{L}_{2}(\mathbf{T})$ and from strong closeness of the set $U$ in $\mathbf{L}_{2}(\mathbf{T})$ (Pironneau, 1984). Let us prove the strong convergence. Let $\left\{\mathbf{u}_{i}\right\}$ be a sequence of functions from the set $U$, minimizing the functional $E(\mathbf{u})$ over $U, \mathbf{u}_{i} \rightarrow \mathbf{u}^{*}$ weakly in $\mathbf{W}_{2}^{1}(\mathbf{T})$ as $i \rightarrow \infty$ and $E\left(\mathbf{u}_{i}\right) \longrightarrow E\left(\mathbf{u}^{*}\right)$ as $i \rightarrow \infty$. If $\mathbf{u} \in X$ then $(\mathbf{u}-\mathbf{z}) \in \stackrel{\circ}{\mathbf{W}}_{2}^{1}(\mathbf{T})$ and $E(\mathbf{u}-\mathbf{z})=E(\mathbf{u})$. The functional $(E(\mathbf{u}))^{1 / 2}$ is equivalent to the standard norm in $\stackrel{\circ}{W}_{2}^{1}(T)$. Weak convergence and convergence of norms imply $\left(\mathbf{u}_{i}-\mathbf{z}\right) \longrightarrow\left(\mathbf{u}^{*}-\mathbf{z}\right)$ strongly in $\stackrel{\circ}{\mathbf{W}}_{2}^{1}(\mathbf{T})$ as $i \rightarrow \infty$. This implies strong convergence of the minimizing sequence in $\mathrm{W}_{2}^{1}(\mathrm{~T})$. 
Remark 2 Let $U_{2}$ be the close of $U_{1}$ in the strong topology of $\mathbf{W}_{2}^{1}(\mathbf{T})$. It is clear that $U_{1} \subset U_{2} \subseteq U . B y$ definitions of $\Phi$ and $U$ from $\mathbf{B} \in \Phi$ it follows that $X(\mathbf{B}) \subset U$ and $\mathbf{u}(\mathbf{B}) \in U$, therefore

$\min _{\mathbf{u} \in U} E(\mathbf{u}) \leq \min _{\mathbf{u} \in U_{2}} E(\mathbf{u})=\min _{\mathbf{u} \in U_{1}} E(\mathbf{u})=\inf _{\mathbf{B} \in \Phi} J(\mathbf{B})$.

\section{Statement 3}

(i) If $U_{\mathbf{2}}=U$ then $\inf _{\mathbf{B} \in \Phi} J(\mathbf{B})=\min _{\mathbf{u} \in U} E(\mathbf{u})$.

(ii) If $U_{2}=U$ then the set of generalized solutions of Problem 1 is equal to the set of solutions of Problem 2.

(iii) If $\inf _{\mathbf{B} \in \Phi} J(\mathbf{B})=\min _{\mathbf{u} \in U} E(\mathbf{u})$ then a generalized solution of Problem 1 is a strong limit point of the corresponding minimizing sequences and is a solution of Problem 2.

\section{Proof.}

(i) Let $\mathbf{u}^{*} \in U$ and $E\left(\mathbf{u}^{*}\right)=\min _{\mathbf{u} \in U} E(\mathbf{u})$. Due to the assumption there exists $\mathbf{B}_{\boldsymbol{i}} \in$ $\Phi$ and $\mathbf{u}_{i} \in X\left(\mathbf{B}_{i}\right)$ such that $\mathbf{u}_{i} \longrightarrow \mathbf{u}^{*}$ strongly in $\mathbf{W}_{2}^{1}(\mathbf{T}) i \rightarrow \infty$. We have $\lim _{i \rightarrow \infty} E\left(\mathbf{u}_{i}\right)=\min _{\mathbf{u} \in U} E(\mathbf{u})$ and $E\left(\mathbf{u}_{i}\right) \geq E\left(\mathbf{u}\left(\mathbf{B}_{i}\right)\right)$, thus inf $\mathbf{B}_{\mathbf{B} \in \Phi} J(\mathbf{B}) \leq \min _{\mathbf{u} \in U} E(\mathbf{u})$. From this and the remark 2 we obtain the equality.

(ii) a) A solution of Problem 2 is a generalized solution of Problem 1. Let $\mathbf{u}^{*} \in$ $U, E\left(\mathbf{u}^{*}\right)=\min _{\mathbf{u} \in U} E(\mathbf{u})$ and let $\mathbf{B}_{\boldsymbol{i}} \in \Phi$ be a sequence of sets and $\mathbf{u}_{i} \in X\left(\mathbf{B}_{\boldsymbol{i}}\right)$ be a sequence of functions such that $\mathbf{u}_{i} \longrightarrow \mathbf{u}^{*}$ strongly in $\mathbf{W}_{2}^{1}(\mathbf{T})$ as $i \rightarrow \infty$. The existence of the sequences follows from the assumption of the statement. It is obvious that $\lim _{i \rightarrow \infty} E\left(\mathbf{u}_{i}\right)=E\left(\mathbf{u}^{*}\right)$. In virtue to remark 1 and the property of solutions of (2) we have $J\left(\mathbf{B}_{i}\right)=E\left(\mathbf{u}\left(\mathbf{B}_{i}\right)\right) \leq E\left(\mathbf{u}_{i}\right)$, therefore $\lim _{i \rightarrow \infty} J\left(\mathbf{B}_{i}\right) \leq E\left(\mathbf{u}^{*}\right)$. But due to remark 2 we have $J\left(\mathbf{B}_{i}\right) \geq E\left(\mathbf{u}^{*}\right)$, therefore

$\lim _{i \rightarrow \infty} J\left(\mathbf{B}_{i}\right)=\lim _{i \rightarrow \infty} E\left(\mathbf{u}\left(\mathbf{B}_{i}\right)\right)=\lim _{i \rightarrow \infty} E\left(\mathbf{u}_{i}\right)=\inf _{\mathbf{B} \in \Phi} J(\mathbf{B})=\min _{\mathbf{u} \in U} E(\mathbf{u})$.

The variational identity (2) implies equality $E\left(\mathbf{u}_{i}\right)-E\left(\mathbf{u}\left(\mathbf{B}_{i}\right)\right)=\| \nabla\left(\mathbf{u}\left(\mathbf{B}_{i}\right)-\right.$ $\left.\mathbf{u}_{i}\right) \mid \mathbf{L}_{2} \|^{2}$. Because the seminorm $\left\|\nabla \mathbf{u} \mid \mathbf{L}_{2}\right\|$ is equivalent to the standard norm in $\stackrel{\circ}{\mathbf{W}}_{2}^{1}(\mathbf{T})$ and $\left(\mathbf{u}\left(\mathbf{B}_{i}\right)-\mathbf{u}_{i}\right) \in \stackrel{\circ}{\mathbf{W}}_{2}^{1}(T)$ we have that $\mathbf{u}\left(\mathbf{B}_{i}\right) \longrightarrow \mathbf{u}^{*}$ strongly in $\mathbf{W}_{2}^{1}(T)$ as $i \rightarrow \infty$ and $\mathbf{u}^{*}$ is a generalized solution of Problem 1 .

(ii) b) A generalized solution of Problem 1 is a solution of Problem 2. Let $\left\{\mathbf{B}_{i}\right\}$ be a minimizing sequence for Problem 1 and $\mathbf{u}\left(\mathbf{B}_{\boldsymbol{i}}\right) \rightarrow \mathbf{u}^{\#}$ weakly in $\mathbf{W}_{2}^{1}(\mathbf{T})$ as $i \rightarrow \infty$, so $\mathbf{u}^{\#}$ is a generalized solution of Problem 1. It follows from $\mathbf{B}_{i} \in \Phi$ that $\mathbf{u}\left(\mathbf{B}_{i}\right) \in U$ and $\mathbf{u}\left(\mathbf{B}_{\boldsymbol{i}}\right) \longrightarrow \mathbf{u}^{\#}$ strongly in $\mathbf{L}_{2}(\mathbf{T})$ as $i \rightarrow \infty$, hence $\mathbf{u}^{\#} \in U$. According to (i) we have

$\lim _{i \rightarrow \infty} E\left(\mathbf{u}\left(\mathbf{B}_{i}\right)\right)=\lim _{i \rightarrow \infty} J\left(\mathbf{B}_{i}\right)=\inf _{\mathbf{B} \in \Phi} J(\mathbf{B})=\min _{\mathbf{u} \in U} E(\mathbf{u}) \leq E\left(\mathbf{u}^{\#}\right)$.

Weak lower semicontinuity of the functional $E$ implies $\lim _{i \rightarrow \infty} E\left(\mathbf{u}\left(\mathbf{B}_{i}\right)\right) \geq E\left(\mathbf{u}^{\#}\right)$, therefore $E\left(\mathbf{u}^{\#}\right)=\min _{\mathbf{u} \in U} E(\mathbf{u})$ and $\mathbf{u}^{\#}$ is a solution of Problem 2 .

(iii) Using the notation of part (ii) b) of the proof we can write

$\inf _{\mathbf{B} \in \Phi} J(\mathbf{B})=\lim _{i \rightarrow \infty} E\left(\mathbf{u}\left(\mathbf{B}_{i}\right)\right) \geq E\left(\mathbf{u}^{\#}\right) \geq \min _{\mathbf{u} \in U} E(\mathbf{u})$, 
hence $\left\|\nabla \mathbf{u}_{i}\left|\mathbf{L}_{2}\|\longrightarrow\| \nabla \mathbf{u}^{\#}\right| \mathbf{L}_{2}\right\|$ as $i \rightarrow \infty$. The strong convergence follows from the weak convergence and from the convergence of norms.

Remark 3 It is known that the close of $\bigcup_{\mathbf{B} \in \Phi} \stackrel{\circ}{\mathrm{W}}{ }_{2}^{1}(\mathbf{T} \backslash \mathbf{B})$ in the strong topology of $\stackrel{\circ}{\mathrm{W}}_{2}^{1}(\mathbf{T})$ equals $\left\{u \in \stackrel{\circ}{\mathrm{W}}_{2}^{1}(\mathbf{T}): \operatorname{meas}(x \in \mathbf{T}: u(x)=0) \geq 1\right\}$ (Suetov, 1994).

\section{A COEFFICIENT OPTIMIZATION PROBLEM}

Let $A>0$. We define the set of admissible coefficients

$$
K_{A}=\left\{k \in L_{\infty}(\mathbf{T}): \int_{\mathbf{T}} k(x) \mathrm{dx} \geq A, \quad \forall x \in \mathbf{T} \quad 0 \leq k(x) \leq A\right\} .
$$

It is obvious that $K_{A}$ is convex and weak compact in $\mathrm{L}_{2}(\mathbf{T})$. For every admissible coefficient $k(x)$ we can consider the boundary value problem

$$
\Delta \mathbf{u}-k \cdot \mathbf{u}=\nabla p, \quad \operatorname{div} \mathbf{u}=0 \quad \text { in } \quad \mathbf{T} ;\left.\quad \mathbf{u}\right|_{\Gamma=\mathbf{z}}=(1,0,0) .
$$

We will denote by $\mathbf{u}(k)$ the weak solution of problem (5).

We define a cost functional on the set of admissible coefficients:

$$
J_{2}(k)=E_{2}(\mathbf{u}(k), k), \quad E_{2}(\mathbf{u}, k)=\int_{\mathrm{T}}\left\{(\nabla \mathbf{u})^{2}+k \cdot \mathbf{u}^{2}\right\} \mathrm{dx} .
$$

Problem 3. It is required to find an admissible coefficient $k_{A} \in K_{A}$ minimizing the functional $J_{2}(k)$ :

$$
J_{2}\left(k_{A}\right)=\min _{k \in K_{A}} J_{2}(k)
$$

Statement 4 Problem 3 has a solution.

Proof. Statement 4 follows from weak compactness of $K_{A}$ and weak continuity of the mapping $k \mapsto \mathbf{u}(k)$.

It is known that $\mathbf{u}(k)$ minimizes the functional $E_{2}(\mathbf{u}, k)$ in the argument $\mathbf{u}$ over the set $X$ (Temam, 1979) :

$E_{2}(\mathbf{u}(k), k)=\min _{\mathbf{u} \in X} E_{2}(\mathbf{u}, k)$.

Remark 4 It is clear from (6) that Problem 3 is equivalent to the minimization problem for the functional $E_{2}(\mathbf{u}, k)$ in both arguments:

$\min _{k \in K_{A}} J_{2}(k)=\min _{\mathbf{u} \in X, k \in K_{A}} E_{2}(\mathbf{u}, k)$. 
Let $h(\mathbf{u})=\sup \{\delta: \operatorname{meas}(x \in \mathbf{T}:|\mathbf{u}(x)| \leq \delta) \leq 1\}$

Theorem 1 Let $k_{A}$ be a solution of Problem $3, \mathbf{u}_{A}=\mathbf{u}\left(k_{A}\right)$. Then

$k_{A}(x)=\left\{\begin{array}{l}A \text { if }\left|\mathbf{u}_{A}(x)\right| \leq h\left(\mathbf{u}_{A}\right), \\ 0 \text { otherwise, }\end{array}\right.$

$\operatorname{meas}\left(x \in \mathbf{T}:\left|\mathbf{u}_{A}(x)\right| \leq h\left(\mathbf{u}_{A}\right)\right)=1, h\left(\mathbf{u}_{A}\right) \longrightarrow 0$ as $A \rightarrow \infty$.

Proof. The proof is based on Remark 4 .

Lemma 2 Let $k_{A}$ be a solution of Problem 3 for every $A>0$ and $\mathrm{u}^{*}$ is a weak limit point of $\left\{\mathbf{u}\left(k_{A}\right)\right\}$ as $A \rightarrow \infty$. Then meas $\left(x \in \mathrm{T}: \mathbf{u}^{*}(x)=0\right) \geq 1$

Theorem 2 Let $k_{A}$ be a solution of Problem 3 for every $A>0$ and $\mathbf{u}^{*}$ is a weak limit point of $\left\{\mathrm{u}\left(k_{A}\right)\right\}$ as $A \rightarrow \infty$. Then $\mathbf{u}^{*}$ is a solution of Problem $2, \mathbf{u}^{*}$ is a strong limit point of $\left\{\mathbf{u}\left(k_{A}\right)\right\}$ and $\lim _{A \rightarrow \infty} J_{2}\left(k_{A}\right)=\min _{\mathbf{u} \in U} E(\mathbf{u})$.

Proof. According to lemma 2 we have $\mathbf{u}^{*} \in U$, therefore it is sufficient to prove that $E\left(\mathbf{u}^{*}\right)=\min _{\mathbf{u} \in U} E(\mathbf{u})$. Let $\mathbf{v} \in U$ and

$\chi_{\mathbf{v}}(x)= \begin{cases}1 & \text { if }|\mathbf{v}(x)|=0 \\ 0 & \text { otherwise }\end{cases}$

Then $A \cdot \chi_{\mathbf{v}} \in K_{A}$ and the following inequalities hold:

$\forall \mathbf{v} \in U \quad E\left(\mathbf{u}\left(k_{A}\right)\right) \leq E_{2}\left(\mathbf{u}\left(k_{A}\right), k_{A}\right)=\min _{\mathbf{u} \in X, k \in K_{A}} E_{2}(\mathbf{u}, k) \leq E_{2}\left(\mathbf{v}, A \cdot \chi_{\mathbf{v}}\right)=E(\mathbf{v})$,

hence $E\left(\mathbf{u}\left(k_{A}\right)\right) \leq E_{2}\left(\mathbf{u}\left(k_{A}\right), k_{A}\right) \leq \min _{\mathbf{u} \in U} E(\mathbf{u})$. Weak lower semicontinuity of the functional $E(\mathbf{u})$ imply the inequalities

$\min _{\mathbf{u} \in U} E(\mathbf{u}) \leq E\left(\mathbf{u}^{*}\right) \leq \liminf _{A \rightarrow \infty} E\left(\mathbf{u}\left(k_{A}\right)\right) \leq \limsup _{A \rightarrow \infty} E\left(\mathbf{u}\left(k_{A}\right)\right) \leq \min _{\mathbf{u} \in U} E(\mathbf{u})$.

Thus $E\left(\mathbf{u}^{*}\right)=\lim _{A \rightarrow \infty} E\left(\mathbf{u}\left(k_{A}\right)\right)=\lim _{A \rightarrow \infty} E_{2}\left(\mathbf{u}\left(k_{A}\right), k_{A}\right)=\min _{\mathbf{u} \in U} E(\mathbf{u})$ and $\mathbf{u}^{*}$ is a solution of the Problem 2 . The strong convergence follows from the weak convergence and from the convergence of norms (see the proof of statement 3 ).

\section{A SHAPE OPTIMIZATION ALGORITHM}

Theorems 1 and 2 suggest the following method for computation of a suboptimal shape:

1) choosing a large number $A$;

2) solving Problem 3 , let $k_{A}$ is a solution, $\mathbf{u}_{A}=\mathbf{u}\left(k_{A}\right)$;

3) taking a set $\mathbf{B}_{A}=\left\{x \in \mathbf{T}:\left|\mathbf{u}_{A}(x)\right| \leq h\left(\mathbf{u}_{A}\right)\right\}$ as a suboptimal shape.

Let me recall connections between Problem 1 and Problem 3. If the hypothesis the close of $\bigcup_{\mathbf{B} \in \Phi} X(\mathbf{B})$ in the strong topology of $\mathbf{W}_{2}^{1}(\mathbf{T})$ is equal to $U$ 
is true then from statement 3 and theorem 2 it follows that limit points of a family $\left\{\mathbf{u}\left(k_{A}\right)\right\}$ are generalized solutions of Problem 1.

Without any hypothesis we can use the following a posteriori argument. It is clear that $J_{2}\left(k_{A}\right)<\min _{\mathbf{u} \in U} E(\mathbf{u}) \leq \inf _{\mathbf{B} \in U} \leq J\left(\mathbf{B}_{A}\right)$ (see the proof of theorem 2), hence inequalities $0<J\left(\mathbf{B}_{A}\right)-\inf _{\mathbf{B} \in U} J(\mathbf{B}) \leq J\left(\mathbf{B}_{A}\right)-\min _{\mathbf{u} \in U} E(\mathbf{u}) \leq\left|J\left(\mathbf{B}_{A}\right)-J_{2}\left(k_{A}\right)\right|+$ $\left|J_{2}\left(k_{A}\right)-\min _{\mathbf{u} \in U} E(\mathbf{u})\right|$ are true. Due to theorem $2 \lim _{A \rightarrow \infty}\left|J_{2}\left(k_{A}\right)-\min _{\mathbf{u} \in U} E(\mathbf{u})\right|=0$. Therefore if the parameter $A$ is big enough and the value $\left|J\left(\mathbf{B}_{A}\right)-J_{2}\left(k_{A}\right)\right|$ is small then $J\left(\mathbf{B}_{A}\right)$ is close to the optimal value $\inf _{\mathbf{B} \in U} J(\mathbf{B})$.

It is obvious that Problem 3 is non-convex. We describe an algorithm for computation of a function that satisfies to a necessary condition of optimality for Problem 3. Let

$\kappa_{-}(x ; \mathbf{u})= \begin{cases}1 & \text { if }|\mathbf{u}(x)|<h(\mathbf{u}), \\ 0 & \text { otherwise }\end{cases}$

and for every $\mathbf{u} \in X$ let $\chi(x ; \mathbf{u})$ be a function such that

$\forall x \in \mathbf{T} \quad \kappa_{-}(x ; \mathbf{u}) \leq \chi(x ; \mathbf{u}) \leq \kappa(x ; \mathbf{u}), \quad \int_{\mathbf{T}} \chi(x ; u) \mathrm{dx}=1$.

We note that $A \cdot \chi(\mathbf{u}) \in K_{A}$.

Theorem 3 Let $k_{0} \in K_{A}, \mathbf{u}_{i}=\mathbf{u}\left(k_{i}\right), k_{i+1}=A \cdot \chi\left(\mathbf{u}_{i}\right)$ and a subsequence $\left\{k_{i_{j}}\right\}$ converges to $k_{*}$ weakly in $\mathrm{L}_{2}(\mathrm{~T})$. Then the sequence $\left\{\mathbf{u}_{i j}\right\}$ converges to a function $\mathbf{u}_{*}$ strongly in $\mathbf{W}_{2}^{1}(\mathbf{T}), \mathbf{u}_{*}=\mathrm{u}\left(k_{*}\right)$ and

$k_{*}(x)=\left\{\begin{array}{lll}A & \text { if } & \left|\mathbf{u}_{*}(x)\right|<h\left(\mathbf{u}_{*}\right) \\ 0 & \text { if } & \left|\mathbf{u}_{*}(x)\right|>h\left(\mathbf{u}_{*}\right)\end{array}\right.$

therefore $\left(\mathbf{u}_{*}, k_{*}\right)$ is a critical point of $E_{2}(\mathbf{u}, k)$ on $X \times K_{A}$.

\section{CONCLUSION}

In this work an algorithm for solving of a shape optimization problem is proposed. An important feature of the algorithm is that the corresponding boundary value problems can be solved on the same domain and grid, with only distinction in the lowest term coefficients of equations. The idea of the algorithm can be applied to other shape optimization problems for minimization of the energy functional of a system.

\section{REFERENCES}

Adams R. (1975) Sobolev spaces. Acad.Press, New York.

Banichuk, N.V. (1983) Problems and methods of optimal structural design. Plenum Press, New York.

YU.S.Osipov, A.P.Suetov (1984) On a problem of J.-L.Lions Soviet Math. Dokl., Vol. 29, No 3 , p.487-91. 
Suetov, A. (1994) A shape optimization algorithm for an elliptic system Control and Cybernetics, 23, No 3, 565-74.

Pironneau, O. (1984) Optimal shape Design for Elliptic Systems. Springer-Verlag, NewYork.

Pironneau O. (1973) On optimal profile in Stokes flow. J. Fluid Mech. 59, part 1, 117-28.

Temam R. (1979) Navier-Stokes equations. Theory and numerical analysis. North-Holland Publishing Company, Amsterdam.

Watson S. R. (1971) Toward the Minimum Drag on a Body of Given Volume in Slow Viscous Flow. J. Inst. Math. Applics 7, No 3, 367-376. 